\title{
Association of State-Issued Mask Mandates with COVID-19 Case and Death Counts
}

\author{
Josh Mitteldorf*, PhD, Madhava Setty**, MD \\ *aging.advice@gmail.com \\ **klmadhava@gmail.com
}

\begin{abstract}
We have interpreted and extended an analysis published on the US CDC website 5 March 2021, which calculated the benefits of imposed mask mandates. Historical studies of masking in the general population have produced contradictory results with respect to their effectiveness in stemming spread of respiratory viral infections. The CDC study is based on a comparison of Daily Growth Rate (DGR) in number of COVID cases (or deaths) before and after imposition of mask mandates. We show that the downward trend in DGR began before masking and continued after masking. We add controls to the CDC methodology by comparing states that imposed mask mandates to states that did not. We report evidence of short-term benefit for the first 30 days that is lost when DGR increased in the fall more in masked states than unmasked. Overall, we find that the benefits reported by CDC can be completely explained by saturation effects, weather changes, and other factors apart from the government mandates that the study was designed to evaluate.
\end{abstract}

\section{Introduction}

On March 5, 2021 the CDC issued a Morbidity and Mortality Weekly report titled "Association of State-Issued Mask Mandates and Allowing On-Premises Restaurant Dining with County-Level COVID-19 Case and Death Growth Rates - United States, March 1-December 31, 2020" [1]. In it the authors claim that "Mask mandates were associated with a 0.5 percentage point decrease $(p=0.02)$ in daily COVID-19 case growth rates $1-20$ days after implementation and decreases of $1.1,1.5,1.7$, and 1.8 percentage points $21-40,41-60,61-80$, and $81-100$ days, respectively, after implementation." Although these percentages are small numbers, they compound daily, so that their long-term effects, if real, would have a substantial impact upon the number of cumulative cases.

We demonstrate that on average, the rate of new COVID cases continued to increase and to increase at an accelerating rate even after mask mandates were imposed. These were not the measures that the CDC report chose to evaluate. Rather, their target measure was an (exponential) Daily Growth Rate (DGR) defined as "the difference between the natural log of cumulative cases or deaths on a given day and the natural log of cumulative cases or deaths on the previous day, multiplied by $100 . "$

DGR was evaluated using linear regression techniques for the 20-day period immediately prior to imposition of mandates. This was defined as the "reference period", which is different in each county. The reference periods from 2,313 US counties were combined to create a composite, 
weighted reference period that represented county-wide DGR prior to mask mandates in those locales.

Seven 20 day periods were chosen for the analysis: two 20-day periods prior to the reference period and five periods following mask mandates. Again, these were combined with weighted average across 2,313 counties. The reported result of the study is that composite DGR during the two periods before the reference period were not significantly different from the reference period, but after mask mandates, DGR declined steadily for five consecutive 20-day periods (Figure 1).

The only controls in this study were the two 20-day periods prior to the reference period. The study did not compare results to states where no mask mandates were imposed.

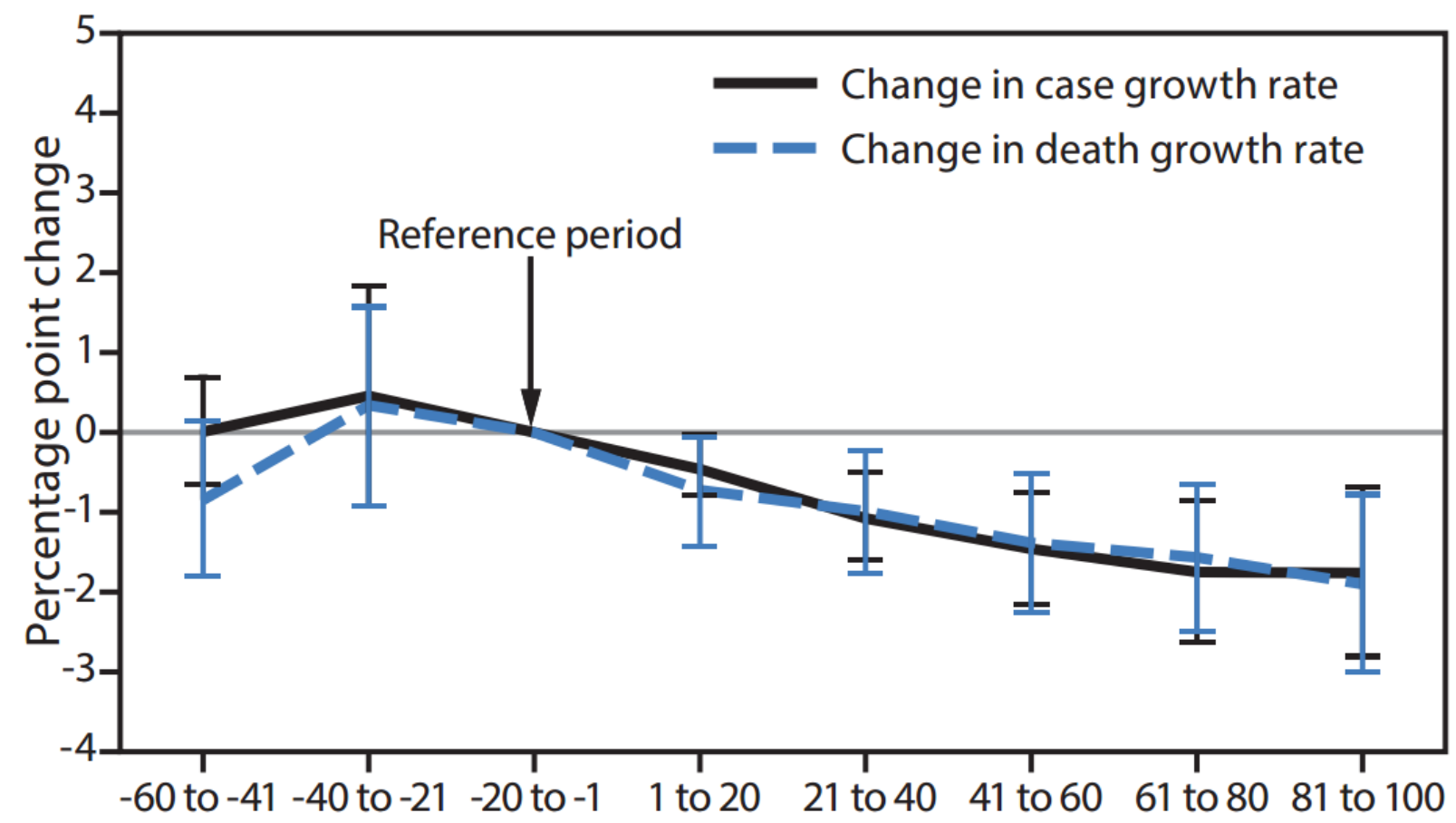

Figure 1 (from Guy et al, ref.\#1) Change in daily case growth rate is continuous at the point of the mask mandate, with no inflection. N.B.The axis label may be confusing. The $Y$ axis is actually Daily Growth Rate, percent change per day, averaged over each 20-day period, except that the 0 is offset to the value of the reference period (days -20 to 1). Guy et al do not include the absolute calibration of the axis,but based on our own attempt to reproduce their work, we estimate the offset is about $2 \frac{1}{2} \%$ per day.

\section{Was masking responsible for the decline in DGR?}

In a viral or bacterial culture, exponential growth occurs early in the culture period, when there is an excess of nutrients and space to grow. Similarly, in the early stage of an epidemic, with many susceptible people and few or no immunes, the number of cases expands exponentially, as in (Figure 2). $\boldsymbol{R}_{\boldsymbol{o}}$ is greater than one, indicating that each infected person, on average, transmits the infection to more than one other person. 


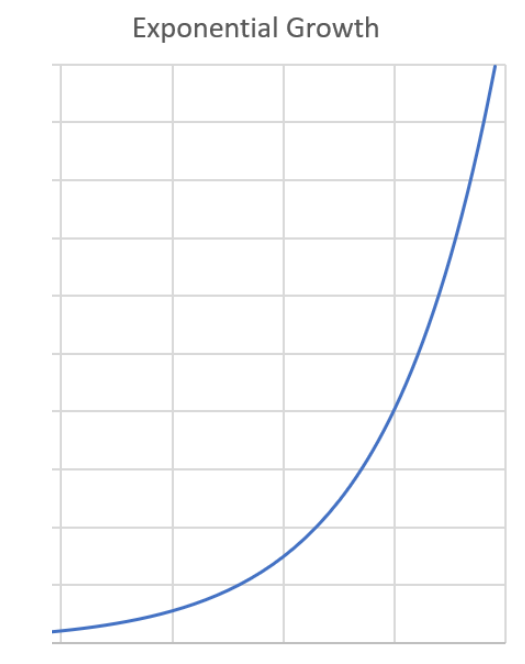

Figure 2 Shape of an exponential curve

However, in nature, no exponential growth can continue for very long. The curve will become less steep, and $\boldsymbol{R}$ at later times is always less than $\boldsymbol{R}_{\mathbf{0}}$. Eventually, each infected person finds less than one vulnerable person to infect, so that $\boldsymbol{R}$ declines below unity, and the epidemic dies out.

This happens for several reasons. First, the most vulnerable people in the community catch the virus early, while the uninfected people that remain may have more pre-existing resistance. Second, people who are exposed very gradually may develop resistance. Third, the infected individuals recover with resistance, so the vulnerable portion of the population decreases. For seasonal respiratory viruses, warmer weather with more humidity is another reason that cases drop. All these effects develop in small, local pockets where the viral outbreak originated, and population viscosity [2] then becomes another factor in the rate of spread.

Therefore, it is expected that the exponential part of the curve will not last very long before the curve begins to flatten. This results in a typical self-limiting growth curve (Figure 3).

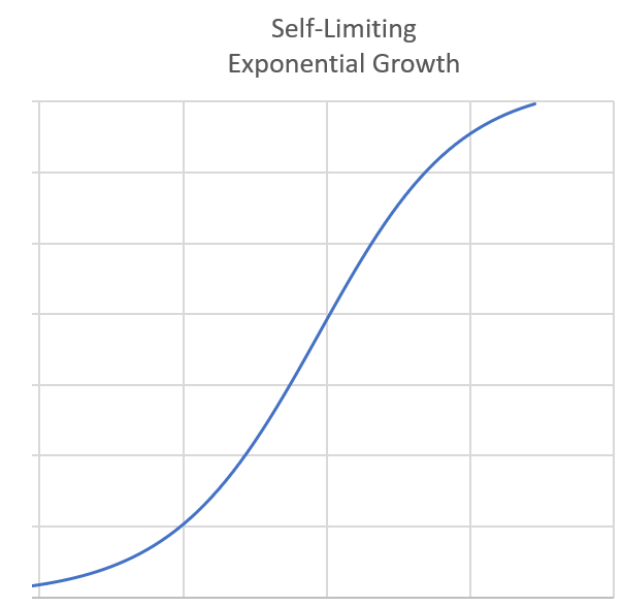

Figure 3 
Indeed, this characteristic curve is demonstrated by the CDC's COVID-19 cumulative case count over time for the United States as a whole (Figure 4). In fact the cumulative case count appears to be two self-limiting exponential growth curves in succession. The first occurred between the beginning of April 2020 and the beginning of October; the second phase began in the Fall when seasonal infectivity induced a second wave of infections.

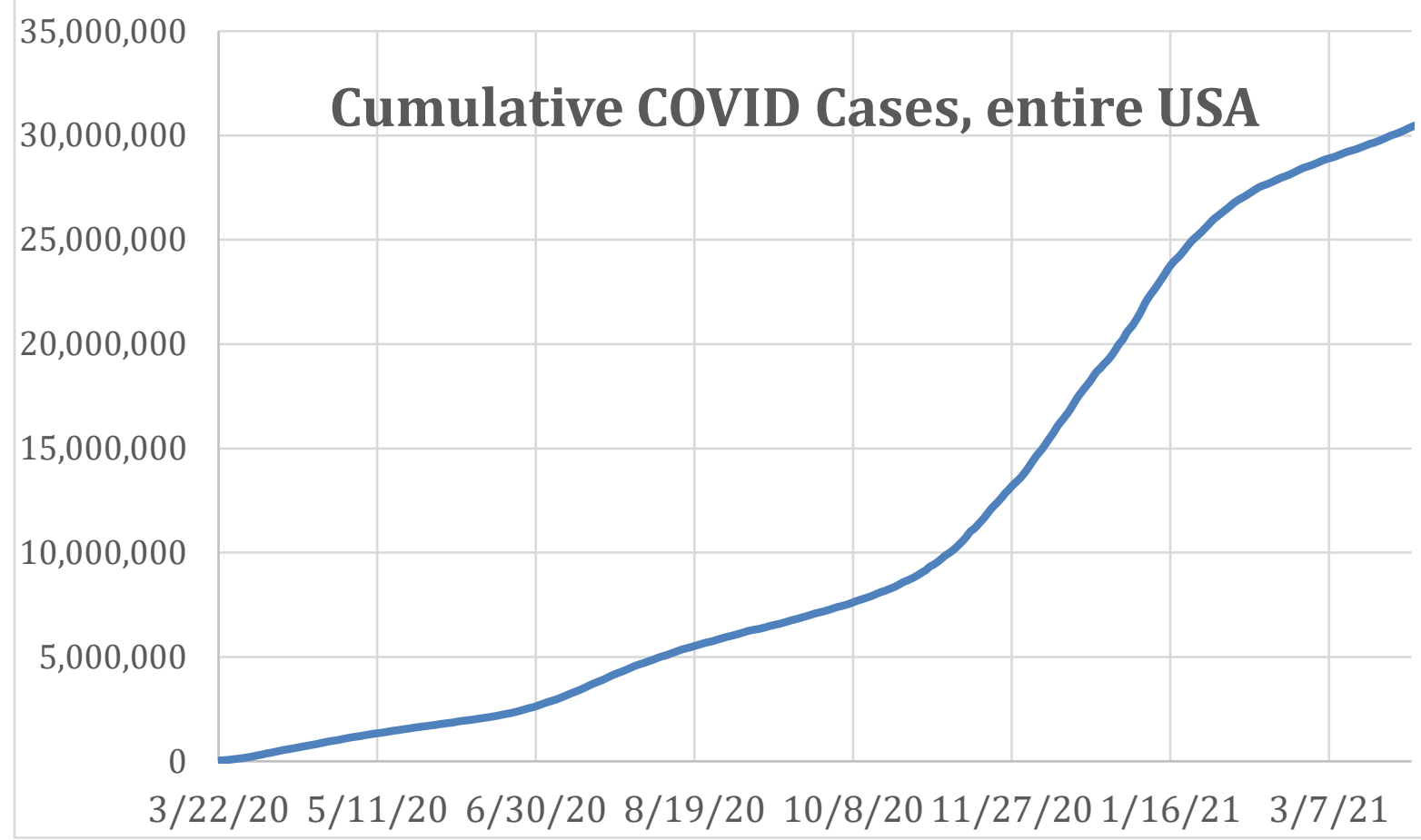

Figure 4 The actual growth in cumulative COVID cases in America [Source=CDC]

Using the definition of Guy et al, Daily Growth Rate for the country appears in Figure 5. 


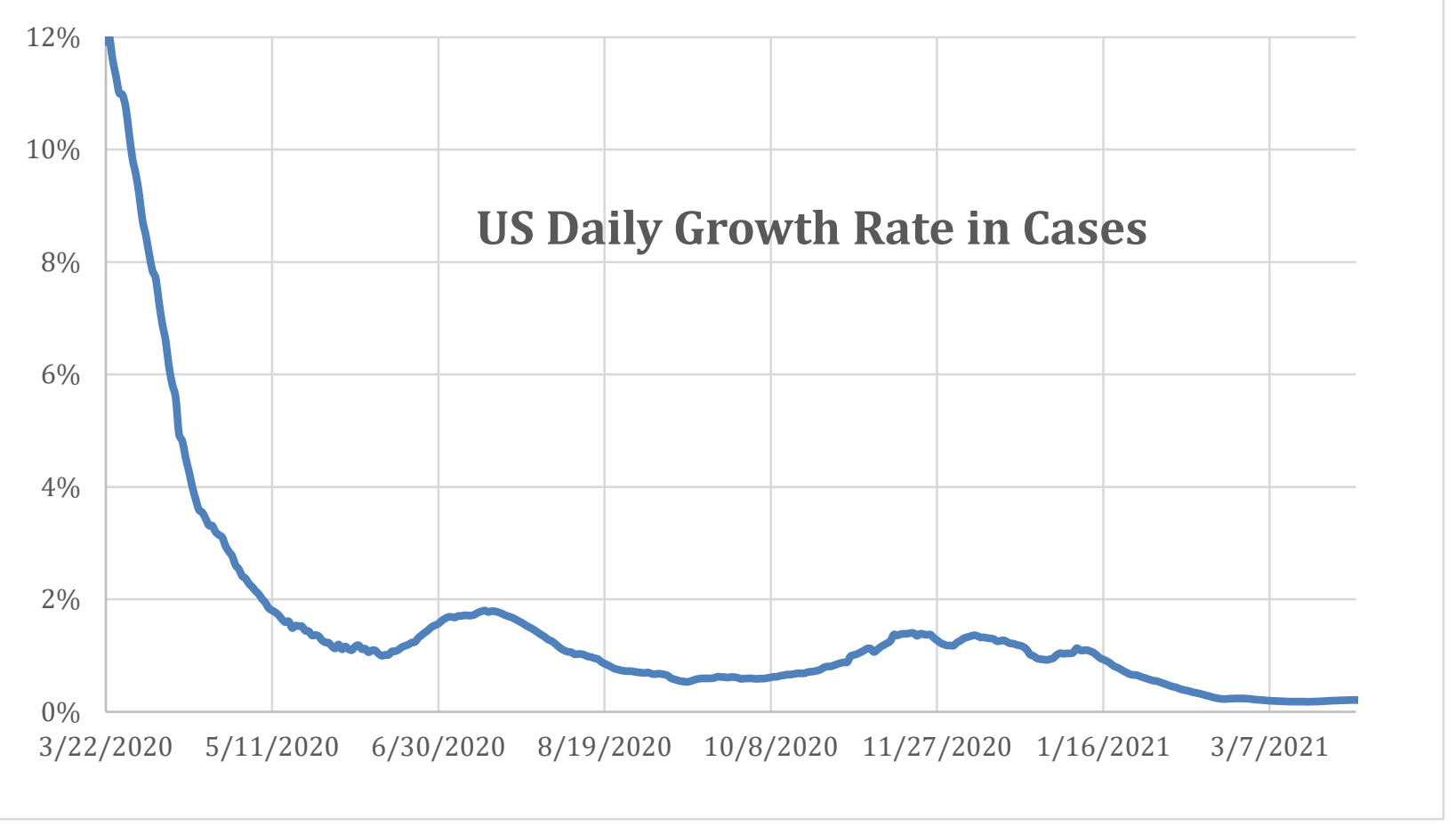

Figure 5 Daily Growth Rate in US COVID cases. There is a long-term decline, characteristic of any epidemic. The decline is punctuated by a "summer bump" when the disease spread to new, uninfected areas, and there was a second, seasonal bump corresponding to the onset of winter weather.

In Figure 5 we see a long-term decline, characteristic of any epidemic. The decline is punctuated by a "summer bump" when the disease spreads to new, uninfected areas, and there is a second, seasonal increase corresponding to the onset of winter weather.

This long-term downward trend is expected in any epidemic and does not necessarily reflect the effects of government-imposed controls. Indeed, we can see a similar curve when DGR is plotted for states in which there were no government mandates (Figure 6). The summer increase is larger, but the fall increase is actually smaller than for regions with mask mandates. 


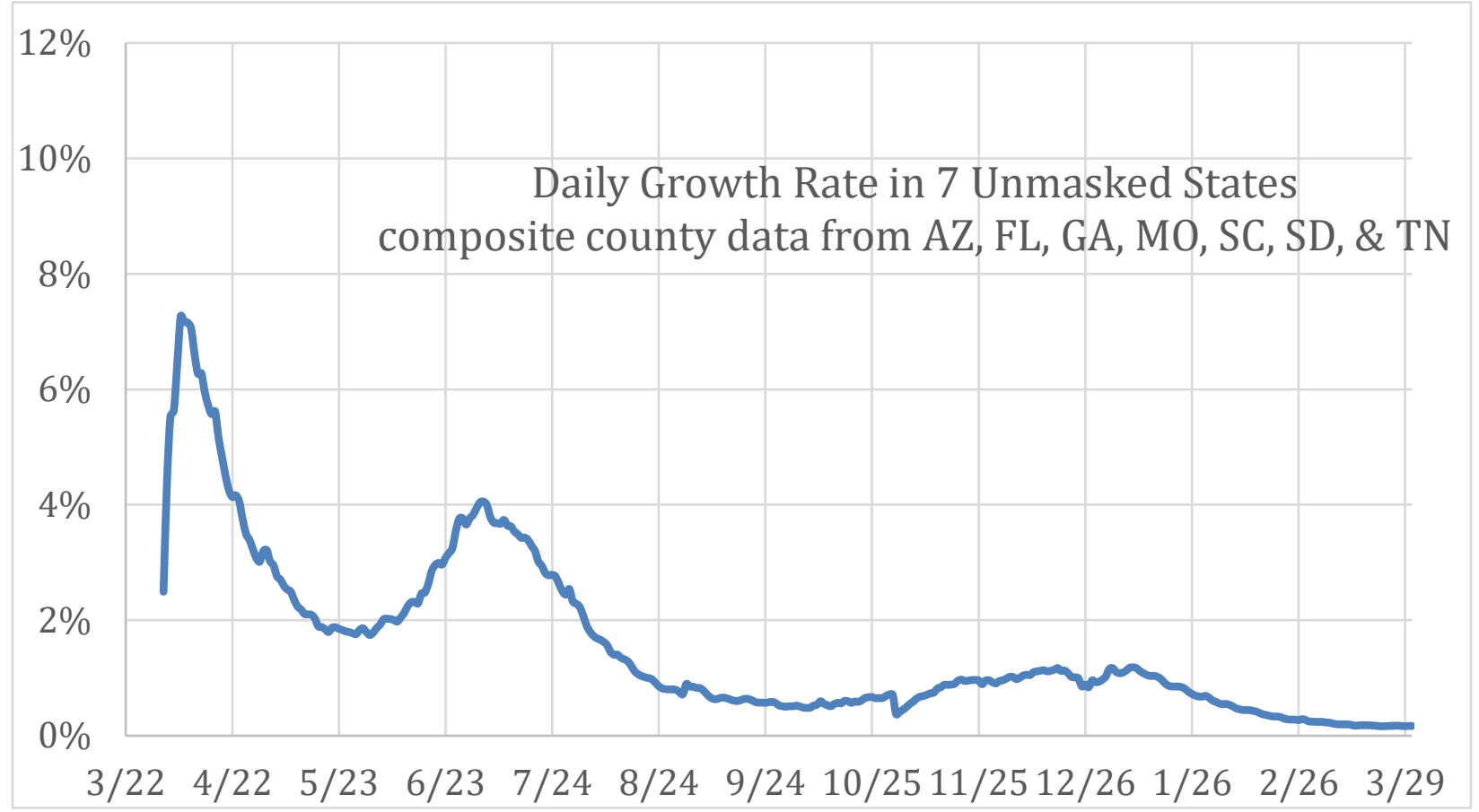

Figure 6 Daily Growth Rate in the 7 most populous states that never imposed mask mandates. The shape of the curve is similar to the masked part of the country. The summer bump is larger, but the fall bump is actually smaller than for regions with mask mandates.

\section{The CDC study uses earlier pre-masking data as a control period}

The plot in Figure 1 is taken directly from the CDC study. It represents their primary result with regard to masking. If we instead examine data from states that remained unmasked throughout the period of observation we arrive at results that are remarkably similar (Figure 7). 


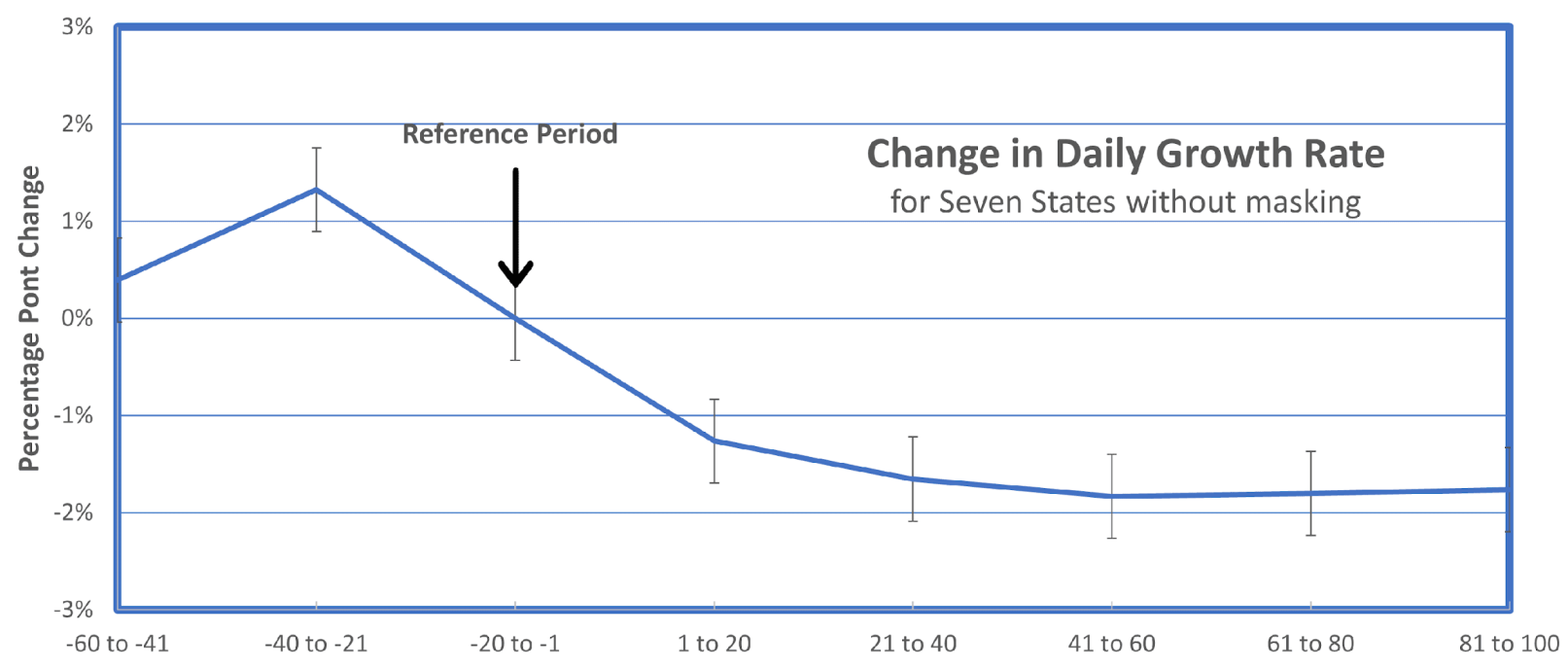

Figure 7 This figure was prepared in the same way as Figure 1, from Guy et al, except that there were no mask mandates in the 7 states covered by this data. The Reference Period was arbitrarily taken ending August 6, 2020.

We have just argued that self-limiting growth always flattens, and therefore we expect a steady decrease in daily growth rate, as defined in the CDC paper. They claim that the two periods before the reference period show no significant trend in DGR, while the five periods after mask mandates show a steady downward trend. Why, then, did they not see a decline in daily growth rate from the periods before the reference period? The answer is that, indeed, they did see a decline from the later control period into the reference period. The decline was the same $0.5 \%$ per 20 days that they observed during two 20-day periods subsequent to mask mandates.

It was not reported as such because the error bars were too wide. Much of the country had not experienced a first wave of cases 40 days before mask mandates were suggested by Federal health officials. The same trend was present, but numbers were too small to support statistical significance. For the period beginning 60 days before most mask mandates (approximately May 2020), the numbers were much smaller still, so the leftmost data point in Figure 1 may indicate only a lack of data pre-pandemic.

California (Figure 8) provides a good counterpoint to the seven unmasked states, because strict lockdowns and masking were mandated during the summer (June 18, 2020). In California, exponential growth in cases continued after mask mandates for a full month at a rate higher than before the mandate. This summer "bump" in DGR was smaller than in the unmasked states, however this difference was offset by a larger fall bump in DGR, as compared to the unmasked states (Figure 6). 


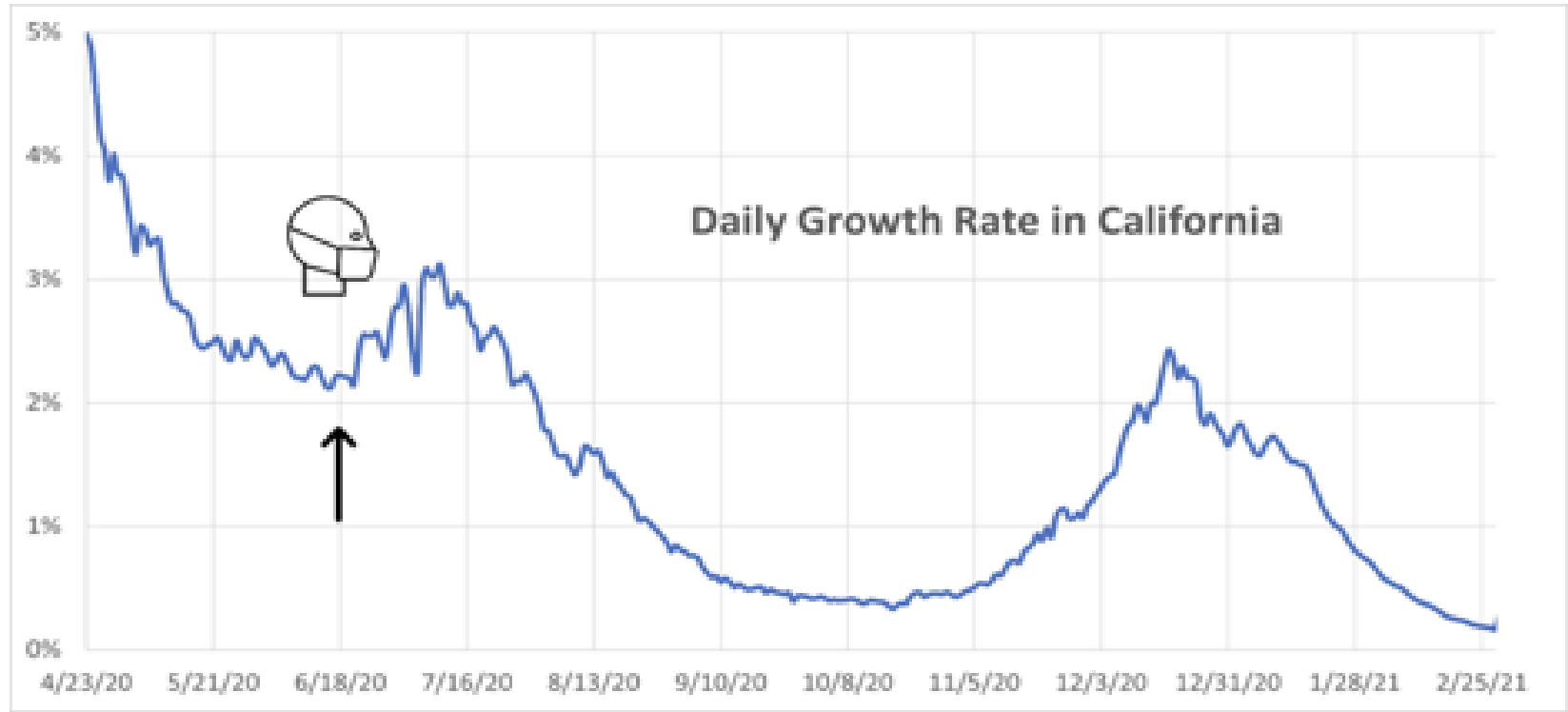

Figure 8 Daily Growth in California cases continued for a month after masking at a rate higher than before masking. This bump was smaller, however, in comparison to the seven unmasked states (Figure 6). In the fall, the relationship was reversed; unmasked states had a smaller second wave compared to California, which remained under mask mandate throughout this period.

\section{Additional Comments on the CDC Methodology}

The CDC report's definition of DGR is the log ratio of cumulative cases on a given day divided by the cumulative reported cases from the previous day $(\operatorname{In}(A)-\ln (B)=\ln (A / B))$. Using all previous cases as the denominator assures that the denominator will always increase as time goes on, which tends to make the curve trend downward unless case growth continues exponentially. This downward trend is a statistical artifact, but it is interpreted in the CDC paper as a decrease attributed to masking policies.

The period considered in the study stops arbitrarily at 100 days, largely excluding the seasonal surge in new cases because the majority of mask mandates were imposed prior to August 2020. Even though the study was conducted (and data downloaded) in February, 2021, it does not include the time period November through January, when masking was still enforced but DGR was high, comparable to the reference period. Had the study included the fall and winter months, the plot in Figure 1 would have extended to a period where the curve stopped declining and rose, as in Figure 5.

\section{Adding Controls to the CDC Study}

The CDC study uses pre-mandate data as a control against which to compare daily growth rates. The problem they encounter, as we have seen, is that data from that time was thin. Is there a better way to determine how much of the decline in exponential growth rate was 
attributable to masking? The question can be addressed by comparing growth of cumulative cases in states that mandated masks with results from similar states in which there were no mandates. Such comparisons are, of course, subject to the caveat that climate, population density, average age and incidence of comorbidities may confound results; nevertheless, even with only approximate comparability, these controls are informative.

We have compared the growth and saturation of COVID cases, comparing pairs of states having similar demographics and climate, one of which mandated masking.while the other did not. Two pairs of states suitable for comparison have been identified: California vs Florida and South Dakota vs North Dakota. Mask mandates were initiated at different times in CA and ND (6/18/2020 and 11/14/2020 respectively). In each paired control state, no mask mandates were imposed.

\section{California vs. Florida}

California imposed mask mandates on 6/18/2020; Florida has not to date mandated masking. Florida compares to California with a similarly mild climate, but has greater population density and higher urbanicity, along with a far higher proportion of geriatric residents ( $21 \%$ vs $15 \%$ over age 65). Both states had similar disease incidence at the time that California imposed its mandate ( 1 case in $242 \mathrm{CA}$ vs 1 case in 260 for FL). Figure 9 demonstrates the growth of cases per $100 \mathrm{~K}$ residents in CA and FL from the time of mask mandate implementation in CA through the end of March, 2021

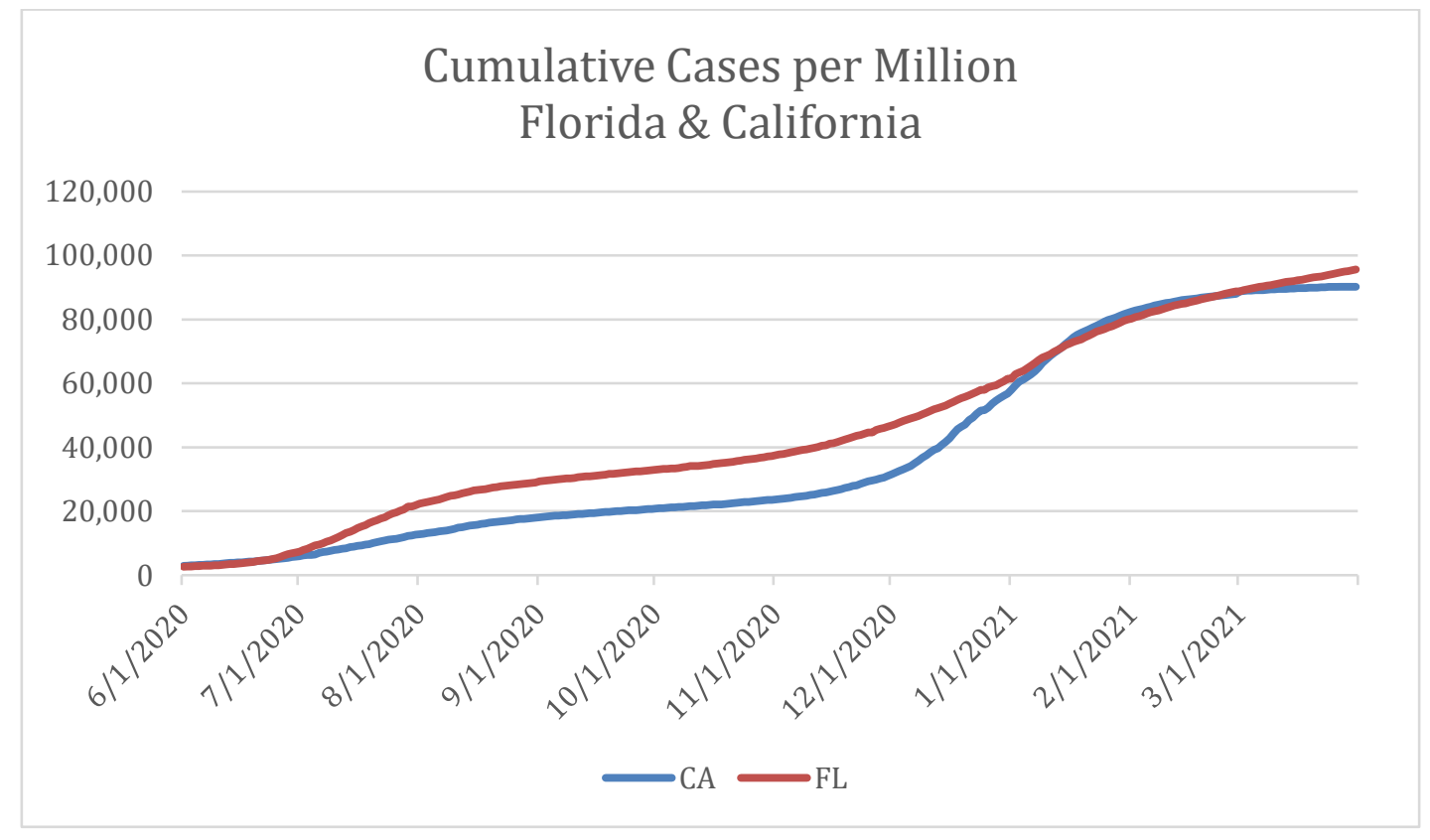

Figure 9 Comparison of California (mask mandate) with Florida

Both states evince two successive phases of self-limiting exponential growth, with the second phase beginning about the end of September. California had a lower per capita burden of new cases during the first epidemic phase. Despite continuing its mask mandates and lockdown, California experienced a rise in cases more severe than Florida during its second wave, so that 
the curves of case growth converged as the second wave was subsiding in February, 2021. This demonstrates that a claimed association between mask mandates and reduced cases is difficult to support.

\section{North Dakota vs. South Dakota}

North Dakota issued a statewide mask mandate on 11/14/2020. At the time, North Dakota had a slightly higher per capita burden of cases than South Dakota. There was little difference between their progressions as case counts in both states more than doubled during the ensuing winter season (Figure 10). Currently, both states are among those with the highest rate of diagnosed cases per person in the US. Mask mandates have not made any overall difference in the number of cases.

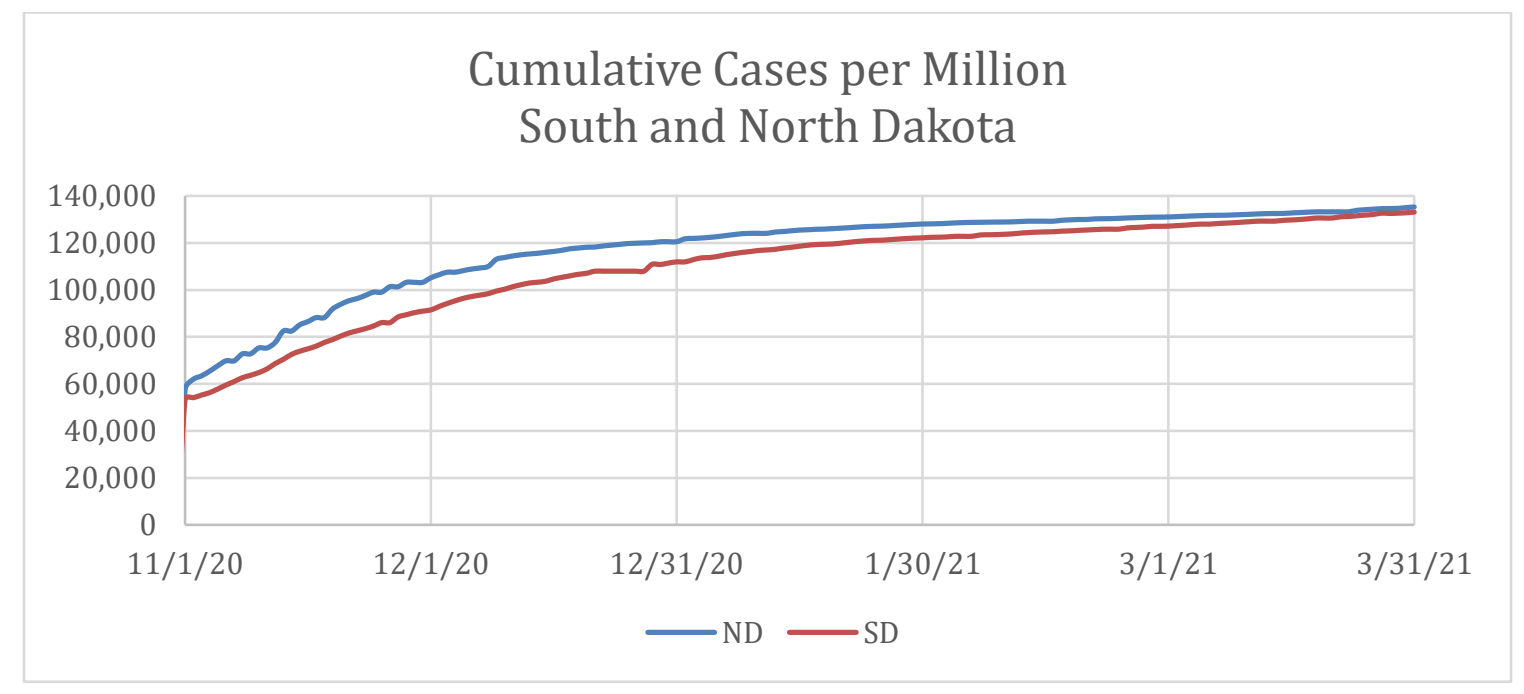

Figure 10 Comparison of North Dakota (mask mandate) with South Dakota

\section{Discussion}

We have identified weaknesses in the methodology of Guy et al that may have contributed to an overestimate of the benefits of government mask mandates in slowing spread of the COVID pandemic.

The increase in incidence of a respiratory borne illness over time, in a given population, is dependent upon various factors, including population density, climate, population age and general health. An initial period of exponential spread of a novel pathogen is expected, and exponential increases in the number of new cases has been observed historically at early stages of other epidemics. With or without medical interventions, exponential growth is invariably tempered, beginning when a minority of the population has been affected. The most vulnerable segments of the population have been depleted, and the disease spreads more 
slowly to subgroups with progressively more resistance. Mathematically, this is seen as the decline in $\boldsymbol{R}$ from an initial value $\boldsymbol{R}_{\boldsymbol{o}}$ to subsequent values which are always less than $\boldsymbol{R}_{\boldsymbol{0}}$.

This steady decline in the exponential growth curve is intrinsic to its epidemiological dynamics and should not be confused with an effect of interventions. In the present case, seasonal patterns of respiratory infectivity must also be considered. Looking at two pairs of comparable states, we have extended the CDC analysis by controlling for saturation and seasonal effects.

In our examination by county of daily cumulative cases of Covid-19, using data extracted from the CDC's (publicly-available) National Environmental Public Health database [3], we were able to identify two pairs of states where reasonable comparisons between a mask vs no mask mandate could be made. In each pair, the state with no mask mandates was more densely populated, yet suffered essentially the same burden of disease as their masked counterpart.

Our investigation parallels a recent study comparing European countries with severe lockdown and masking restrictions to other countries with looser restrictions. Bjørnskov found no evidence that the restrictions affected COVID case rates, consistent with our US comparisons [4].

We find that the benefits that CDC attributes to lockdowns and masking can largely be explained by saturation effects and seasonality.

\section{REFERENCES}

1. Guy Jr, G.P., et al., Association of State-Issued Mask Mandates and Allowing OnPremises Restaurant Dining with County-Level COVID-19 Case and Death Growth Rates_United States, March 1-December 31, 2020. Morbidity and Mortality Weekly Report, 2021. 70(10): p. 350.

2. Pollock, G.B., Population viscosity and kin selection. American Naturalist, 1983: p. 817829.

3. $\quad$ CDC, National Environmental Public Health Tracking Network. 2021.

4. Bjørnskov, C., Did Lockdown Work? An Economist's Cross-Country Comparison. An Economist's Cross-Country Comparison (August 2, 2020), 2020. 